\title{
Considering Human and Organizational Factors in Risk Industries
}

\section{What Should We Expect? How Do We Manage this Subject?}

\section{Christian Neveu, Valérie Lagrange, Philippe Noël and Nicolas Herchin}

\begin{abstract}
The industrial partners of the Foundation for an industrial safety culture (FonCSI) agree on the importance of considering human and organizational factors (HOF) for safety. Nevertheless, many questions remain regarding how to address this issue in industrial organizations. In this short chapter, HOF experts of companies supporting FonCSI and representing various industrial sectors (energy, transports, oil \& gas) expose their viewpoint on HOF goals, strategies, approaches, methods and tools.
\end{abstract}

Keywords Risk management $\cdot \mathrm{HOF}$ approach $\cdot$ Safety strategy

\section{HOF Approach: Features and Benefits}

Since the creation of the risk industries, different kinds of approaches have been developed in order to avoid accidents: although at first purely technical, since the 1980s these approaches have taken into account human and organizational factors (HOF) (see Fig. 1).

Today, the lessons learned from each of these historical steps show that risk industries need to implement a HOF approach with particular features:

- an integrated approach, distinct from the "man or machine" dichotomy;

C. Neveu ( $\varangle)$

SNCF, Paris-Saint-Denis, France

e-mail: christian.neveu@laposte.net

V. Lagrange

EDF, Paris-Saint-Denis, France

P. Noël

TOTAL, Paris-La Défense, France

N. Herchin

GRTgaz, Paris-Saint-Denis, France 


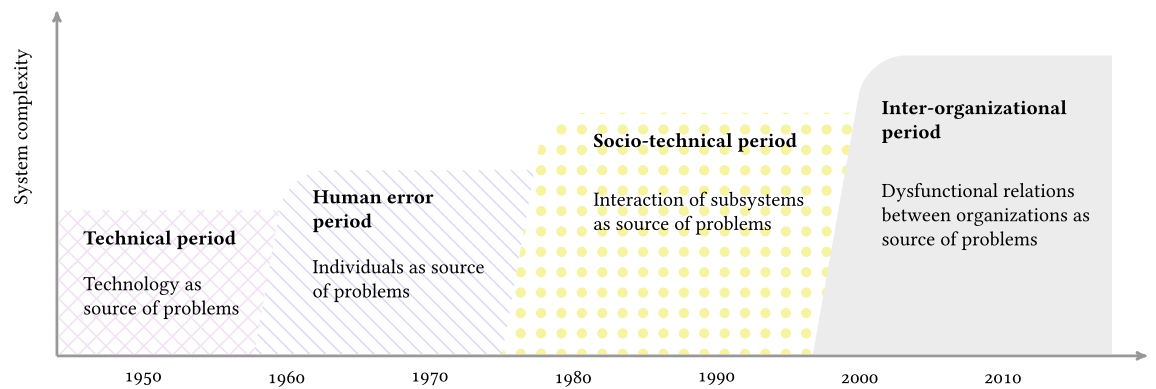

Fig. 1 Evolution of risk management approaches (adapted from [1, 2])

- a realistic vision of the 'human', as a factor of reliability with limits, whose role must be facilitated by:

- reducing the situations favourable to errors and failures,

- reinforcing the capacity and the means to manage the diversity and the unforeseen nature of operational situations;

- a "field" approach, based on the interactions between technical systems, humans and organization in real situations.

For these reasons, FonCSI's definition of HOF seems to be particularly pertinent: "identify and set up the conditions which favour a positive contribution of operators and collectives in safety".

Furthermore, with this kind of HOF approach, even if it focuses on safety and security performances, it actually contributes more globally to the quality of operation as a whole and is positive for all performances.

Following these historical steps, such programs initially dealt with human factors in incident investigation, ergonomics in the workplace and human factors in design. Then, the influences of sociology and psychology led to research to understand problems in organizations that resulted in incidents. The issues of decision making, safety culture, management of change, cooperation, are now also taken into consideration. They concern everybody, from the operator to the senior vice-president and all managers; they concern corrective actions after incidents, but are increasingly focused on preventive actions, which form part of the initial training for individuals and of continuous improvement action plans for units (Fig. 2). 


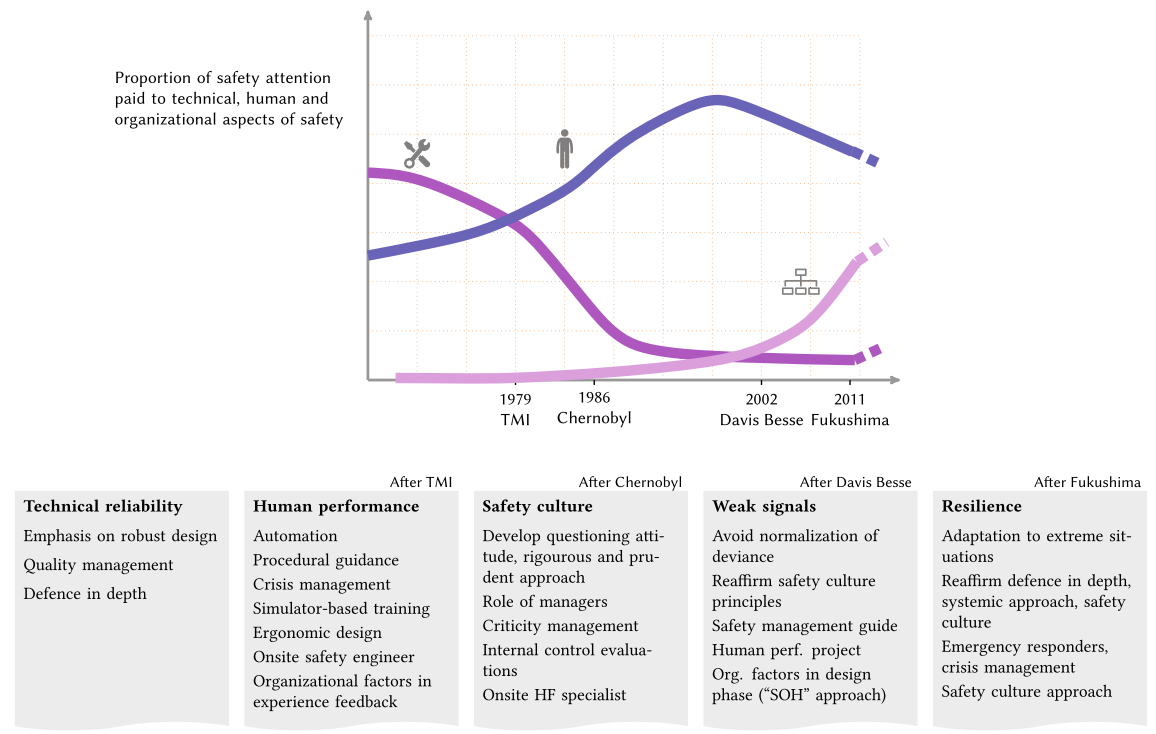

Fig. 2 Evolution of the attention given to the dimensions of safety management at EDF (adapted, with permission, from an internal EDF document)

\section{How Do We Implement and Manage HOF Approaches?}

Through the historical development of HOF approaches in risk industries-aeronautics, railways, nuclear, oil \& gas... and now in the health sector, an "ideal" implementation has clearly emerged:

- It would begin by creating a team of $H O F$ Experts $^{1}$ at the corporate level, in order to define a roadmap (aims and strategy for the next 3-4 years, based on a diagnosis) addressed to the top management and to suggest methods and tools for supporting the HOF dynamics.

- Then, it needs to benefit from relays in the organization, by putting in place $H O F$ consultants $^{2}$ or HOF correspondents ${ }^{3}$ at local levels or intermediate levels in charge of developing action plans: HOF training, field analysis, support departments and local managers for main actions such as implementing HOF methods and tools such as incident investigation, human performance, operational decision making ... (Fig. 3).

\footnotetext{
${ }^{1}$ Expert means Ph.D. or at least 3rd cycle graduate in ergonomics, psychology, or sociology, with a position in the organization in order to support the top management in HOF domain.

${ }^{2}$ Consultant means a $4-5$ years mission carried out by a person who may have an operational background but has received additional high-level training in HOF.

${ }^{3}$ Correspondent means a person well trained on one specific HOF method (short training), identified as a referent inside her/his department.
} 

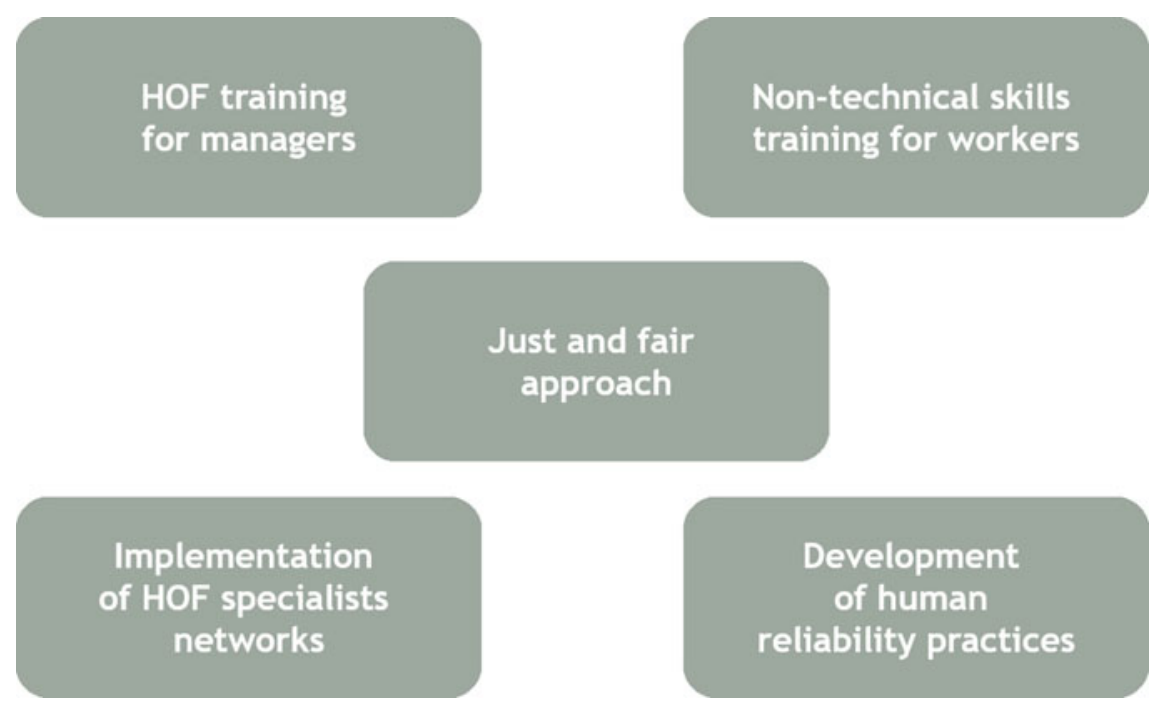

Fig. 3 Content of the SNCF HOF project

Depending on the current challenges of the company and its maturity level in this area, the implementation of the HOF approach could take various forms. Nevertheless, three invariants need to be respected:

- Whatever the methods used by companies, the effectiveness of the program relies on an in-depth understanding of functions and risks, discovering the real human activity in a job or a task. It must be focused on what people do, how safety is produced, what can go wrong and what can be done to prevent this.

- In each sector, the HOF approach must be controlled: by the safety management system (SMS), with a clear ambition, action plan, measure of performance and efficiency.

- The basis of HOF dynamics requires competencies, from experts/consultants/correspondents, but also from each employee, including managers. Everybody has to acquire HOF skills—-knowledge and know-how-in order to develop a safety culture and good practices.

\section{Difficulties and Opportunities}

The first key problem to achieving the objectives of HOF programs observed in companies is the turnover of staff in the management line, and specifically at the senior management level. The senior management of the entity must be involved in the program as the main sponsor. This is a key condition to ensuring that it will be taken to its conclusion even though the environment may change. However, each 
time the senior management changes, the program runs the risk of being stopped or reinitialized, with a period of questioning. Sometimes the turnover rate is so high that the outcomes of such program are lost.

Another aspect is the continuous moving environment. The worldwide or domestic market can quickly change because of unforeseen circumstances; this could result in a decision being made to change the organization, the methods implemented and/or the tools. Because HOF programs need time to achieve their results, in a moving environment they are exposed to be defined again in order to be well-adapted.

It probably means that HOF are not really integrated enough in safety management processes. They depend to a large extent on the conviction of individuals, not of the whole organization.

The second key problem observed on how HOF programs work is the impact of the regulator which has not necessarily reached a high level of maturity in the HOF approach (e.g. looking for responsibility versus discovering the work situation). Based on how the observed reaction of the regulator is perceived, the HOF program could be influenced.

Moreover, most of the HOF programs that have been performed in companies are mainly focused on the human factors that can influence human performance: inadequate procedures, inadequate communication, inability to implement lessons learned, perceived lack of management and commitment to safety. It is still a challenge to really implement programs addressing organizational factors with inputs from sociology. When we analyse some recent big changes in organization (at corporate or site level), it is clear that companies still have to improve their ability to analyse organizational factors and customize their change program based on the results of such analyses.

Training is one of the ways of making progress. Training must cover all the employees of the organization, from the executive management to frontline workers. When most of the HOF training is integrated in the baseline competencies training program, efficiency is better. It facilitates the incorporation of HOF methods and behaviours in daily management and operational tasks. With this practice, managers strive to consider HOF methods as a further step to managing the organization, broader than safety. Frontline workers are more convinced that HOF influence their safety decisions and actions, and thus more likely to deliver safe acts.

One challenge for companies is to gather information on the tools, methods and studies that have been used with success, with the relevant agility in a moving environment. They could be established as a reference for some questions or problems that were an issue in the past and have resurfaced in another branch of the company, or sometimes, unfortunately, in the same branch.

A collection of good practices can also be made between companies, whether they are from the same sector or not. 


\section{As a Conclusion}

According to the benchmark of the different risk industries and to the international experts' points of view, ${ }^{4}$ we can say that a HOF approach cannot be reduced to the use of methods and tools. At the core of such an approach, a strong conviction from the top managers of the organization is required, expressed by a clear ambition (i.e. strategic vision and required means) and a strong commitment that is visible to everybody in daily decisions and behaviours.

\section{References}

1. J. Reason, Managing the management risk: New approaches to organisational safety, in Reliability and Safety in Hazardous Work Systems: Approaches to Analysis and Design, ed. by B. Wilpert, T. Qvale (Lawrence, Hove, 1993), pp. 7-21

2. B. Wilpert, B. Fahlbruch, Safety related interventions in interorganisational fields, in Safety Management and the Challenge of Organisational Change, ed. by A. Hale, M. Baram (Elsevier, Oxford, 1998)

Open Access This chapter is licensed under the terms of the Creative Commons Attribution 4.0 International License (http://creativecommons.org/licenses/by/4.0/), which permits use, sharing, adaptation, distribution and reproduction in any medium or format, as long as you give appropriate credit to the original author(s) and the source, provide a link to the Creative Commons license and indicate if changes were made.

The images or other third party material in this chapter are included in the chapter's Creative Commons license, unless indicated otherwise in a credit line to the material. If material is not included in the chapter's Creative Commons license and your intended use is not permitted by statutory regulation or exceeds the permitted use, you will need to obtain permission directly from the copyright holder.

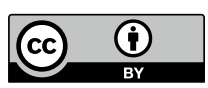

\footnotetext{
${ }^{4}$ The experts that participate in the FonCSI residential seminar held in January 2018 (Editor's note).
} 